\title{
P-0344 - Clinical improvement of dapagliflozin in patients with prediabetes and overweight/obesity
}

\section{González-Ortiz, A.M. Ramírez-Rodríguez, E. Martínez-Abundis, C. Covarrubias-Martínez.}

Institute of Experimental and Clinical Therapeutics, University of Guadalajara; Guadalajara, México.

\section{Background}

The selective and reversible inhibition of SGLT2 by dapagliflozin, which reduces renal reabsorption of glucose and promotes its excretion through urine, has demonstrated clinical benefits in patients with T2DM, however, these findings in prediabetes and overweight/obesity are unknown.

\section{Aim}

The aim of this study was to evaluate the clinical effects of dapagliflozin in patients with prediabetes and overweight/obesity.

\section{Materials and Methods}

\section{Design:}

Randomized, double-blind, placebocontrolled clinical trial.

\section{Subjects:}

Twenty-four patients of both sexes between 30 and 60 years of age, with diagnosis of prediabetes (IFG + IGT) and overweight/obesity, without pharmacological treatment.

\section{Pharmacological intervention:}

Subjects were randomly assigned to two groups of 12 patients each, to receive 10 $\mathrm{mg}$ of dapagliflozin (Forxiga $\AA^{\circledR}$, Astra Zeneca \& Bristol Meyers) or placebo, orally once a day before breakfast for 12 weeks.

\section{Procedures:}

Clinical measurements (body weight, BMI, waist circumference, body fat and blood pressure) and laboratory measurements (glucose and insulin concentrations at 0, 30, 60,90 and 120 minutes by a $75 \mathrm{~g}$-OGTT, A1C, triglycerides, total cholesterol, HDL-C, LDL-c, VLDL and uric acid concentrations) were evaluated at baseline and at the end of the study, as well as adherence and tolerability were assessed.

\section{Statistical Analysis:}

Data were analyzed with Wilcoxon (intragroups) and Mann-Whitney U (intra-groups) tests. A $p \leq 0.05$ was considered statistically significant.

\section{Ethical considerations:}

Procedures were carried out in accordance with national and international guidelines, including Guidelines for Good Clinical Practice. The protocol was approved by a local Research Ethics Committee.

\section{Results}

All subjects who were eligible after enrollment, completed the 12 weeks of the pharmacological intervention, including 9 females and 3 males in the placebo group and 8 females and 4 males in the dapagliflozin group. The mean age of patients was $46.7 \pm 9.8$ and $51.5 \pm 6.3$ years in the placebo and dapagliflozin group respectively, without significant difference between groups.

There were no significant differences between groups at baseline.

There were a significant decrease in fasting plasma glucose, uric acid, body, BMI, waist circumference and body fat after dapagliflozin administration. There were no significant differences after placebo administration (Table 1).

IFG was improved in $100 \%$ of patients treated with dapagliflozin vs. $25 \%$ in placebo group, whereas an improvement in IGT was observed in $42 \%$ and $33 \%$ of patients treated with dapaglifozin and placebo respectively (Figure 1).

Table 1. Characteristics before and after pharmacological intervention

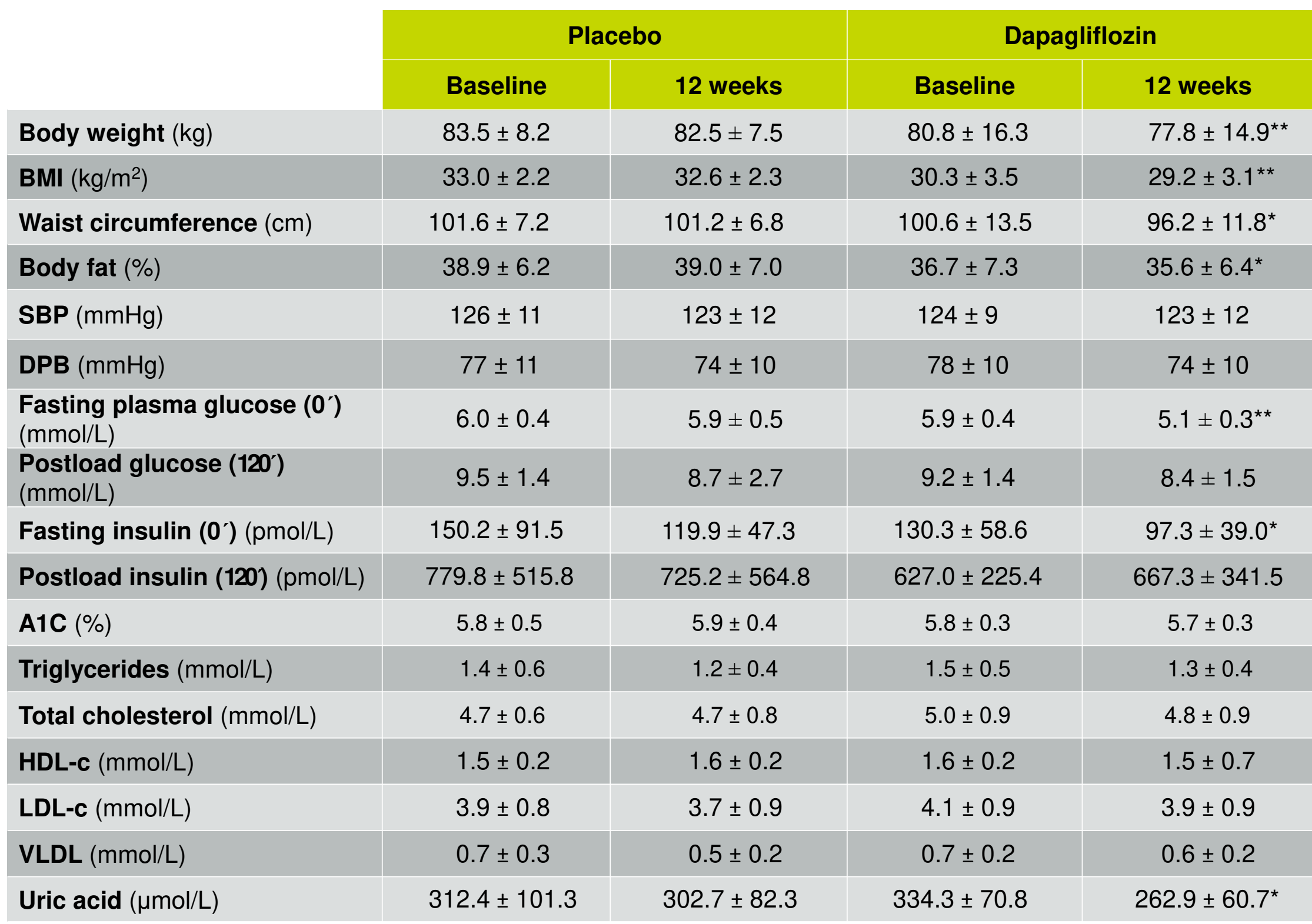

Data are expressed as mean and standard deviation.

Significant differences baseline vs. 12 weeks by group: * $p<0.05,{ }^{* *} p<0.005$ ( $p$ value obtained by Wilcoxon rank test).

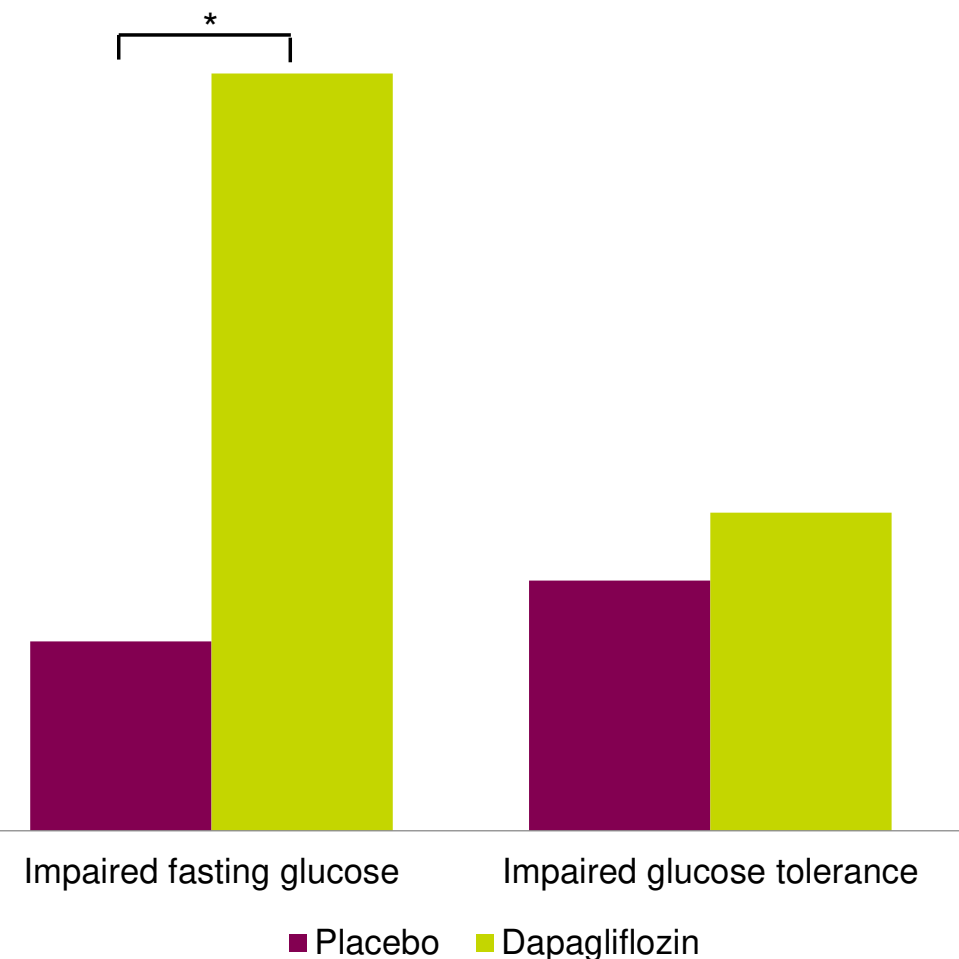

Placebo Dapagliflozin

Data are expressed as percentage.

Significant differences placebo vs. dapaglilozin : * $p<0.05$

Figure 1. Percentage of remission of prediabetes

\section{Conclusion}

Dapagliflozin administration during 12 weeks improved impaired fasting glucose and decreased fasting plasma glucose, fasting insulin, uric acid and all anthropometric measurements.

\section{Abbreviations}

A1C (glycated hemoglobin A1c), BMI (body mass index), DBP (diastolic blood pressure), HDL-c (high density lipoprotein cholesterol), IFG (Impaired fasting glucose), IGT (impaired glucose tolerance), LDL-C (low density lipoprotein cholesterol), OGTT (oral glucose tolerance test), SBP (systolic blood pressure), SGLT2 (sodium-glucose cotransporter type 2), T2DM (Type 2 Diabetes mellitus), VLDL (very-low density lipoprotein). 